Sebastian Jeworutzki \& Jörg-Peter Schräpler

\title{
Kleinräumiges regionales Bildungsmonitoring - Analysen mit amtlichen Zensus- und Schuldaten in Nordrhein-Westfalen
}

\section{Zusammenfassung}

Der Beitrag zeigt anhand einer umfassenden Längsschnittstudie für Nordrhein-Westfalen Möglichkeiten für ein kleinräumiges regionales Bildungsmonitoring mit amtlichen Daten auf (Jeworutzki et al., 2017). Im Rahmen der Analysen werden der langfristige wirtschaftliche und soziodemographische Strukturwandel unterhalb der Gemeindeebene untersucht und Zusammenhänge mit räumlichen Bildungsdisparitäten analysiert. Dazu werden typische strukturelle Entwicklungen in den Untersuchungsräumen identifiziert und der Einfluss der Entwicklungsmuster auf die Höhe der Übergangsquote von der Grundschule zum Gymnasium diskutiert. Datengrundlage für die Analysen sind einerseits amtliche Daten auf Einzelschulebene für den Zeitraum 2003 bis 2015 und andererseits Daten der Volkszählungen 1961, 1970, 1987 und 2011, die für kleinräumige zeitinvariante Bezirke (Stadtteilebene) aufbereitet wurden.

Schlüsselwörter: Regionales Bildungsmonitoring, Bildungsdisparitäten, Zensusdaten, Gymnasialquote

\section{Monitoring Education with Small-Area Official Statistics in North Rhine-Westphalia}

\begin{abstract}
Based on a comprehensive longitudinal study for North Rhine-Westphalia, the article shows possibilities for small-scale regional educational monitoring with official data (Jeworutzki et al., 2017). We examine the long-term economic and socio-demographic structural change below the municipal level and analyze correlations with spatial educational disparities. For this purpose, typical structural developments in the study areas are identified and the influence of development patterns on the level of transition from elementary to grammar school is discussed. Data for the analyses are on the one hand official data at individual school level for the period 2003 to 2015 and on the oth-
\end{abstract}


er hand data of the census 1961, 1970, 1987 and 2011 which were prepared for smallscale time-invariant districts (district level).

Keywords: regional educational monitoring, educational disparities, census data, transition to grammar school

\section{Einleitung}

Im Rahmen der Sozialberichterstattung wird unter Monitoring das systematische Erfassen, Messen, Beobachten und Überwachen von gesellschaftlichen Vorgängen und Prozessen, die als relevant angesehen werden, verstanden. Hierbei spielt insbesondere die wiederholte, regelmäßige, systematische Durchführung eine zentrale Rolle, um Bezüge und Veränderungen adäquat zu protokollieren und die entsprechenden Daten auswerten zu können. In diesem Sinne ist das Monitoring eine wichtige gesamtgesellschaftliche Aufgabe, deren Ziel es ist, über gesellschaftliche Strukturen und Prozesse $\mathrm{zu}$ informieren, so dass auf deren Grundlage gesellschaftspolitische Maßnahmen geplant und begründet werden können (vgl. bspw. NRW.ProjektSoziales GmbH, 2017, S. 9).

In Anlehnung an die schon seit vielen Jahren praktizierte Sozialberichterstattung des Bundes, der Länder und vieler Kommunen, die sich schwerpunktmäßig mit dem Thema „Armut“ und der Lebenslage von in Armut lebenden Personen und Bevölkerungsgruppen beschäftigen, wurden inzwischen in unterschiedlichen zeitlichen Abständen auch Bildungsberichte veröffentlicht. Von der Kultusministerkonferenz wurde im Jahr 2006 eine Gesamtstrategie zum Bildungsmonitoring beschlossen (KMK, 2016). Neben der regelmäßigen Teilnahme an internationalen Leistungsstudien und der Überprüfung von Bildungsstandards wird hier vor allem die regelmäßige Bildungsberichterstattung als wesentliches Instrument hervorgehoben. Als Herausforderung und zentrale Aufgabe der Bildungspolitik werden dabei sowohl die Erhöhung der Bildungsqualität insgesamt als auch die gleichzeitige Verbesserung der Bildungschancen für alle Schüler*innen angesehen (ebd., S. 5).

In den Bildungsberichten werden üblicherweise auch die gesellschaftlichen Rahmenbedingungen mit dargestellt (vgl. exemplarisch Autorengruppe Bildungsberichterstattung, 2018). In einer Vielzahl von Studien (z. B. Peisert, 1967; Fickermann, 1999; Terpoorten, 2014; Baumert et al., 2010) konnte gezeigt werden, dass kleinräumige sozialstrukturelle und sozialräumliche Bedingungen einen maßgeblichen Einfluss auf die Bildungsteilhabe und Bildungschancen der Bevölkerung haben und als Kontexte miteinbezogen werden müssen. Allerdings bleibt die Erfassung des Kontextes in den bisherigen Bildungsberichten hinter den Möglichkeiten zurück: Erstens beziehen sich die Bildungsberichte des Bundes oder der Länder nur auf die jeweilige administrative Gliederung. Regionale Bildungsberichte wie etwa in Nordrhein-Westfalen 
der Bildungsbericht Ruhr (Regionalverband Ruhr, 2012) verbleiben in ihrer räumlichen Betrachtung zumeist auf einer Kreis- oder Gemeindeebene. Die Heterogenität der Lebensbedingungen innerhalb der Städte wird hier kaum mitberücksichtigt. Im Falle der kommunalen Bildungsberichte ist dann zwar i. d. R. eine kleinräumige Darstellung gegeben; hier fehlt jedoch aufgrund der Beschränkung auf die jeweilige Einzelkommune eine notwendige übergreifende Perspektive.

Ein regionales Bildungsmonitoring ist insbesondere immer dann relevant, wenn zwischen den beteiligten Kommunen enge strukturelle Verbindungen und stabile Austauschbeziehungen bestehen. Isolierte kommunale Betrachtungen greifen dann zu kurz. Dies lässt sich in Nordrhein-Westfalen (NRW) sehr gut anhand des Ruhrgebietes $^{1}$ zeigen. Die strukturellen und demografischen Besonderheiten der Region hängen mit ihrer langen industriellen Prägung durch Kohle und Stahl zusammen. In den großen Städten überlagern sich soziale, ethnische und demografische Ungleichheiten, wobei sich Armut in den stark vom Bergbau geprägten nördlichen Stadtteilen der Städte konzentriert. Insofern ist auch eine kleinräumige Analyse unterhalb der Gemeindeebene notwendig.

Für das Ruhrgebiet konnte anschaulich gezeigt werden (Jeworutzki, Knüttel, Niemand, Schmidt, Schräpler \& Terpoorten, 2017; Jeworutzki \& Knüttel, 2018), dass in vielen Fällen verfestigte Bildungsnetzwerke existieren: Die Schülerströme zwischen Grundschulen und weiterführenden Schulen machen nicht an den jeweiligen kommunalen Grenzen halt. Zudem sind wichtige sozialstrukturelle Entwicklungen zumeist nicht auf eine Kommune beschränkt, sondern sind regionale Phänomene. Empfehlenswert ist somit ein kleinräumiges regionales oder sogar überregionales Bildungsmonitoring, welches zudem auch Einblick in die bisherige Entwicklung der Bildungslandschaft bietet.

In den nachfolgenden Abschnitten wird anhand von Ergebnissen aus der Längsschnittstudie Wege zur Metropole Ruhr (Schräpler, Jeworutzki, Butzin, Terpoorten, Goebel \& Wagner, 2017) gezeigt, wie auf Basis von kleinräumigen amtlichen Sozialraum- und Schuldaten ein flächendeckendes kleinräumiges Bildungsmonitoring umgesetzt werden kann. Das hier dokumentierte Vorgehen ist nicht speziell auf die Datenverfügbarkeit in NRW beschränkt, sondern lässt sich prinzipiell mit aktuellen Daten auch deutschlandweit umsetzen. Als Grundlage dienen neben den amtlichen Schuldaten auf Schulebene, die Bestandteil jeder Landesstatistik sind, Zensusdaten,

1 Zum Ruhrgebiet zählen die Städte Bottrop, Bochum, Dortmund, Duisburg, Essen, Gelsenkirchen, Hagen, Hamm, Herne, Mülheim an der Ruhr, Oberhausen und die Landkreise Ennepe-Ruhr, Recklinghausen, Unna und Wesel. Die Städte und Kreise sind zum Regionalverband Ruhr (RVR) zusammengeschlossen. 
die zumindest seit dem Zensus 2011 für alle Bundesländer auch kleinräumig auf Rasterebene unterhalb der Gemeindeebene verfügbar sind. ${ }^{2}$

In dem hier betrachteten Beispiel stehen auch historische Zensusdaten zur Verfügung, so dass erstmals über eine weiter in die Vergangenheit reichende Längsschnittbetrachtung die sozialstrukturelle Entwicklung von kleinräumigen Bezirken unterhalb der Gemeindeebene für ganz NRW mit dem Fokus auf das Ruhrgebiet analysiert werden kann. Vor diesem Hintergrund werden die Prozesse der Bildungssegregation dargestellt und die Zusammenhänge zwischen residentieller Segregation und kleinräumigen Bildungsdisparitäten analysiert. Das hier beschriebene Vorgehen kann zum einen dazu genutzt werden, handlungsrelevantes Wissen über die Schulen einer Region zu generieren. Zum anderen kann es im Kontext der Stadt(teil)entwicklung klein- und sozialräumliche Informationen zur lokalen Bildungssituation liefern.

Die Analyse der kleinräumigen sozialstrukturellen Entwicklungen des Schulumfeldes und der Schülerzusammensetzung ist nicht auf deskriptive Auswertungen beschränkt, sondern es lassen sich mittels multivariater Verfahren z.B. für einzelne Schulen Erwartungswerte in Bezug auf Output-Indikatoren wie etwa die Übergangsquote zum Gymnasium ableiten. Bezogen auf ihren Sozialraum unerwartet hohe Übergangsquoten machen so auf Schulen aufmerksam, die mit innovativen pädagogischen Konzepten oder unter speziellen Rahmenbedingungen besondere Erfolge erzielen. Diese Erkenntnisse könnten dann in weitere Planungen zur Steigerung der Bildungsqualität miteinbezogen werden.

\section{Datengrundlage}

Für die Analyse der regionalen sozialstrukturellen Disparitäten werden Daten der Volkszählungen 1961, 1970, 1987 und 2011 auf Ebene der Orts- bzw. Stadtteile genutzt. Um räumliche Analysen zu ermöglichen, wurden die Raumabgrenzungen für die Ortsteile der Gemeinden in NRW zum Stand des Jahres 2011 digitalisiert. ${ }^{3}$ Im Rahmen einer Sonderauswertung wurden die Daten des Zensus 2011 diesen Ortsteilen zugespielt. ${ }^{4}$ Die Daten aus den vergangenen Volkszählungen liegen in

2 So werden über den Zensus-Atlas Bevölkerungszahlen für das Jahr 2011 auf einem 100-Meter-Raster und umfassende Personen-, Haushalts- und Familienmerkmale auf einem Quadratkilometer bereitgestellt. Zudem geben die Forschungsdatenzentren der Statistischen Ämter des Bundes und der Länder wissenschaftlichen Einrichtungen weitere Möglichkeiten, kleinräumigen Daten zu analysieren. Der Zugang zu kleinräumigen historischen Daten aus früheren Volkszählungen wird vermutlich nur in einzelnen statistischen Landesämtern möglich sein.

3 Die Abgrenzungen basieren bei Gemeinden mit mehr als 100.000 Einwohnern auf kommunalen Daten. Für die kleineren Gemeinden werden Ortsteilgeometrien der Firma infas 360 genutzt (Jeworutzki et al., 2017, S. $46 \mathrm{ff}$.).

4 Die Daten des Zensus 2011 werden um einige kommerzielle Daten von infas 360 zur Erwerbstätigkeit ergänzt. 
NRW zu den verschiedenen Zeitpunkten zu unterschiedlichen Ortsteilaggregaten vor und mussten manuell den Ortsteilabgrenzungen zugeordnet werden.

Das neue Erhebungsverfahren des Zensus 2011 erlaubt es, kleinräumige Analysen mit den erhobenen Registermerkmalen durchzuführen. Insbesondere für überregionale Analysen steht daher eine kleinräumige Datenquelle mit einem einheitlichen Indikatorenset zur Verfügung, auch wenn aufgrund des langen Aktualisierungsintervalls von zehn Jahren keine kurzfristigen Veränderungen abgebildet werden können. Da aber die sozialen und ökonomischen räumlichen Disparitäten zeitlich relativ beständig sind, können die Daten der Volkszählungen einen wichtigen Beitrag zur Beschreibung des räumlichen Kontextes der Bildungsinstitutionen liefern.

Für Zeitreihenanalysen ist es wichtig, dass die regionale Gliederung über die Zeit hinweg möglichst unverändert bleibt, damit sichergestellt ist, dass Niveauunterschiede in den betrachteten Indikatoren nicht durch Veränderungen im Raumbezug hervorgerufen werden. Für die weiteren Analysen werden daher „zeitinvariante Bezirke“ konstruiert, die im Hinblick auf die Datenverfügbarkeit der Volkszählungen so aus den Ortsteildaten aggregiert werden, dass identische Raumabgrenzungen für die verschiedenen Zeitpunkte entstehen. Für NRW führt das Verfahren zu 1.586 unterschiedlich großen zeitinvarianten Bezirken mit durchschnittlich 11.058 Einwohnern (403 bis 40.764 Einwohner). ${ }^{5}$ Insbesondere in den Gebieten, in denen durch die Gemeindereform neue Strukturen geschaffen wurden, ergeben sich teilweise etwas größere räumliche Aggregate, da sonst eine eindeutige Zuordnung der Ortsteile nicht möglich geworden wäre.

Die Analyse der Bildungsdisparitäten basiert auf Schuleinzeldaten des statistischen Landesamtes NRW (IT.NRW) für die Schuljahre 2003/2004 bis 2014/2015. Der Datensatz umfasst die Zahl der Übergänge zwischen den einzelnen öffentlichen Schulen, differenziert nach Geschlecht und Nationalität. Die Schuldaten wurden georeferenziert, so dass zum einen eine Zuordnung zu den zeitinvarianten Bezirken möglich ist und zum anderen die Luftlinienentfernungen zwischen den Grundschulen und weiterführenden Schulen berechnet werden konnten.

\section{Analyse von Grundschulübergängen zu weiterführenden Schulen}

Im Rahmen der Bildungsberichterstattung und der Diskussion um ungleiche Bildungschancen spielen die Übergangsquoten $\mathrm{zu}$ den weiterführenden Schulen eine zentrale Rolle (Stadt Essen, 2011; Stadt Dortmund, 2014; Landeshauptstadt München, 2016) und werden u. a. als Indikator für die Durchlässigkeit und Selektivität der Bildungsgänge genannt (vgl. Döbert, 2007). In der Studie Wege zur Metropole Ruhr

5 Jeweils das 5. und 95. Perzentil. 
(Schräpler et al., 2017) wurden sowohl die Grundschulübergänge zu weiterführenden Schulen als auch die Schulformempfehlungen für NRW flächendeckend im Längsschnitt analysiert. Wir beschränken uns im Rahmen der Deskription auf den Anteil der Schüler*innen der Grundschulen im Zeitraum 2011 bis 2014, die nach der vierten Klasse auf ein Gymnasium gewechselt haben.

Die Fokussierung auf das Gymnasium soll nicht unterstellen, dass der Besuch eines Gymnasiums der alleinige Indikator für Bildungserfolg ist. Schüler*innen können auch in anderen Schulformen erfolgreiche Bildungskarrieren absolvieren oder auch im weiteren Verlauf ihrer Ausbildung die Schulform wechseln. Gleichzeitig ist jedoch festzustellen, dass der Gymnasialbesuch nicht nur wie in der Vergangenheit eine zentrale Rolle beim Erwerb der Hochschulreife spielt, sondern auch in vielen Ausbildungsberufen ein Abitur vorausgesetzt wird oder dieses Vorteile in der Konkurrenz um Ausbildungsplätze mit sich bringt (vgl. Bundesministerium für Bildung und Forschung, 2017; Baethge, Solga \& Wieck, 2007, S. 40). Da an den Gesamtschulen nur eine Minderheit der Absolvent*innen das Abitur macht, ist die Übergangsquote zur Gesamtschule als Indikator für die Bildungsteilhabe als vergleichsweise schwierig zu bewerten.

Die Übergangsquoten variieren deutlich zwischen den einzelnen Schulen, aber auch zwischen den Regionen (siehe Schräpler et al., 2017, S. 175). Bei den Übergängen zum Gymnasium und zu den Gesamtschulen finden sich Schulen mit extrem niedrigen (unter 2\%) und auch extrem hohen Übergangsquoten (über 95\%). Die Extremwerte sind bei den Übergängen zur Real- und Hauptschule weniger stark ausgeprägt. Betrachtet man das Niveau der Übergangsquoten, dominiert auch auf Einzelschulebene das Gymnasium mit 41,4 Prozent in NRW und mit 39,5 Prozent im Ruhrgebiet. Die durchschnittlichen Übergangsquoten zu den anderen Schulformen sind deutlich geringer und variieren weniger stark. Auffällig ist, dass die durchschnittliche Übergangsquote zur Gesamtschule im Ruhrgebiet mit 28,6 Prozent deutlich höher liegt als in ganz NRW mit 22,3 Prozent und gleichzeitig die durchschnittlichen Übergänge zur Hauptschule geringer ausfallen. Die großen Unterschiede zwischen den Schulen zeigen, dass die eigentlich relevante Analyseebene für die Frage nach der Wechselbeziehung zwischen Sozialstruktur und Bildungsbeteiligung unterhalb der Gemeindeebene liegt (Peisert, 1967; Bartels, 1975; Hauf, 2007; Terpoorten, 2014). Dies sollte auch bei einem flächendeckenden regionalen Bildungsmonitoring mitberücksichtigt werden. Erst dann ist es möglich, die Heterogenität innerhalb der Kommunen adäquat zu analysieren. 


\section{Kontextanalyse: Einfluss des Sozialraums}

\subsection{Typisierung der kleinräumigen Bezirke}

Im Rahmen von Bildungsberichten werden i. d. R. mehrere Dimensionen der Sozialstruktur betrachtet, wobei insbesondere die demographische, ethnische und soziale Segregation im Vordergrund steht (vgl. bspw. Hetmeier, Schneider, Wolf, Klostermann, Gnahs \& Weiß, 2014). Die vorliegende Analyse der kleinräumigen soziostrukturellen Disparitäten berücksichtigt darüber hinaus mehrere Indikatoren aus den Volkszählungen 1970 und 2011. Um typische Unterschiede und Gemeinsamkeiten im Hinblick auf die wirtschaftliche und soziodemografische Entwicklung der betrachteten Gebiete zu erfassen und gleichartige Bezirke zu identifizieren, wird eine Typisierung der Räume durchgeführt.

Als Methode für die Typisierung der kleinräumigen Bezirke in NRW dient die latente Klassenanalyse, ein modernes probabilistisches Klassifikationsverfahren, welches im Gegensatz zu einer traditionellen Clusteranalyse die hierarchische Struktur der Daten mitberücksichtigen und die Wahrscheinlichkeit für eine Klassenzuordnung quantifizieren kann. ${ }^{6}$ Eine detaillierte Beschreibung der verwendeten Indikatoren und eine Darstellung weiterer Einzelergebnisse finden sich in Jeworutzki et al. (2017); hier sollen vor allem die zentralen Ergebnisse skizziert werden. ${ }^{7}$

Insgesamt wurden für die Bezirke in NRW neun Cluster identifiziert (siehe Tab. 1 und Abb. 1). Vier Cluster davon (Cluster 1 bis 4 ) beschreiben vor allem die Heterogenität im ländlichen Raum. Gemeinsam ist diesen Clustern, dass die Bezirke in ihnen neben einer unterdurchschnittlichen Einwohnerdichte im Mittel einen leicht überdurchschnittlichen Jugendquotienten und einen unterdurchschnittlichen Migranten- und Arbeitslosenanteil aufweisen.

Die restlichen fünf Cluster beziehen sich in erster Linie auf Städte bzw. Ballungsgebiete, wobei die Cluster 5, 6 und 7 mehrheitlich im Ruhrgebiet liegen. Diese drei Cluster sind vom Strukturwandel besonders stark betroffen; sie zeigen einen deutlichen Rückgang im Anteil an Beschäftigten im Produzierenden Gewerbe.

Cluster 5 kann als sozialökonomisch konsolidierter Cluster bezeichnet werden. Er beschreibt vor allem Bezirke im südlichen Teil des Ruhrgebiets; es gehören aber

6 Die Bezirke sind auf einer höheren Ebene in Gemeinden eingebettet. Diese „Mehrebenenstruktur" sollte bei einer Typisierung mitberücksichtigt werden.

7 Als Indikatoren dienen auf Ebene der Bezirke der Jugendquotient 2011, die Differenz Jugendquotient 2011 zu 1970, der Migrantenanteil 2011, die Differenz Ausländeranteil 2011 zu 1970, der Leerstand 2011 in Prozent, der Arbeitslosenanteil 2011 in Prozent, der Beschäftigtenanteil im Produzierenden Gewerbe 2011 am Arbeitsort, die Differenz der Beschäftigtenanteile im Produzierenden Gewerbe 2011 zu 1970 in Prozent. 
Tab. 1: Beschreibung der ermittelten neun Cluster

\begin{tabular}{|c|c|c|c|c|c|c|c|c|c|c|}
\hline & \multicolumn{9}{|c|}{ Cluster } & \multirow{2}{*}{ insg. } \\
\hline & 1 & 2 & 3 & 4 & 5 & 6 & 7 & 8 & 9 & \\
\hline Anzahl Bezirke & 271 & 281 & 164 & 170 & 207 & 163 & 145 & 82 & 103 & 1.586 \\
\hline Einwohner*innen (in 1.000) & 5.153 & 1.055 & 1.195 & 661 & 2.776 & 2.096 & 2.296 & 778 & 1521 & 17.538 \\
\hline $\begin{array}{l}\text { Mittelwert Jugendquotient } \\
2011 \text { in \% }\end{array}$ & 31,4 & 27,8 & 32,5 & 30,3 & 25,3 & 23,6 & 30,2 & 26,6 & 20,0 & 28,0 \\
\hline $\begin{array}{l}\text { Differenz des Jugend- } \\
\text { quotienten } 2011 \text { - } 1970 \text { in } \\
\text { Prozentpunkten }\end{array}$ & $-20,5$ & $-33,4$ & $-33,3$ & $-31,1$ & $-22,6$ & $-19,3$ & $-14,4$ & $-16,1$ & $-14,2$ & $-24,2$ \\
\hline $\begin{array}{l}\text { Mittelwert des Migranten- } \\
\text { anteils } 2011 \text { in \% }\end{array}$ & 22,7 & 10,8 & 14,4 & 13,6 & 16,9 & 25,0 & 38,3 & 27,2 & 28,2 & 20,2 \\
\hline $\begin{array}{l}\text { Differenz des Ausländer- } \\
\text { anteils } 2011 \text { - } 1970 \text { in } \\
\text { Prozentpunkten }\end{array}$ & 2,6 & 1,4 & 2,2 & 1,4 & 2,3 & 7,1 & 11,6 & 5,3 & 6,8 & 3,9 \\
\hline $\begin{array}{l}\text { Mittelwert der Leerstands- } \\
\text { quote } 2011 \text { in \% }\end{array}$ & 3,3 & 3,7 & 2,7 & 3,8 & 3,4 & 4,5 & 4,8 & 3,9 & 2,5 & 3,6 \\
\hline $\begin{array}{l}\text { Mittelwert des Anteils der } \\
\text { Erwerbstätigen im Produzie- } \\
\text { renden Gewerbe } 2011 \text { in \% }\end{array}$ & 26,2 & 17,5 & 33,7 & 58,0 & 22,3 & 17,8 & 20,8 & 55,2 & 8,6 & 27,4 \\
\hline $\begin{array}{l}\text { Differenz der Erwerbs- } \\
\text { tätigen im Produzierenden } \\
\text { Gewerbe zwischen } 2011 \\
\text { und } 1970 \text { am Arbeitsort in } \\
\text { Prozentpunkten }\end{array}$ & $-31,1$ & $-32,7$ & $-22,9$ & $-4,0$ & $-30,8$ & $-28,7$ & $-35,0$ & $-8,2$ & $-28,1$ & $-26,4$ \\
\hline $\begin{array}{l}\text { Mittelwert des Anteils der } \\
\text { Erwerbslosen } 2011 \text { in \% }\end{array}$ & 6,2 & 4,4 & 3,9 & 4,6 & 8,2 & 12,2 & 12,9 & 10,1 & 7,2 & 7,2 \\
\hline \multicolumn{11}{|l|}{ Kovariablen } \\
\hline $\begin{array}{l}\text { Mittlere Einwohnerzahl } \\
\text { je } \mathrm{km}^{2}\end{array}$ & 602 & 204 & 174 & 209 & 1295 & 2160 & 1939 & 1808 & 1991 & 975 \\
\hline $\begin{array}{l}\text { Selbstgenutztes Wohn- } \\
\text { eigentum } 2011 \text { in \% }\end{array}$ & 54,8 & 64,3 & 63,8 & 63,6 & 48,4 & 28,1 & 28,5 & 36,8 & 24,9 & 49,5 \\
\hline $\begin{array}{l}\text { Anteil der Gebiete im } \\
\text { Ruhrgebiet in \% }\end{array}$ & 0,6 & 8,7 & 0,0 & 0,0 & 55,1 & 96,0 & 45,4 & 32,1 & 0,0 & 25,2 \\
\hline
\end{tabular}

Quelle: Jeworutzki et al., 2017, S. 114; eigene Berechnungen

auch Räume am nördlichen Rand dazu. Der Cluster umrahmt wie ein Speckgürtel das Ruhrgebiet. Die Arbeitslosenquote liegt zwar etwas über dem Landesschnitt, aber noch deutlich unter dem Ruhrgebiets-Durchschnitt. Die Leerstandsquote, der Migrantenanteil und auch der Zuwachs im Ausländeranteil sind unterdurchschnittlich. Der Jugendquotient weist den stärksten Rückgang auf. Letztlich werden mit Cluster 5 vor allem die wohlhabenderen Bezirke im Ruhrgebiet gekennzeichnet, welche schon immer von Wohn- und Lebensformen des Bürgertums geprägt sind; dies zeigt sich u. a. auch in dem - im Vergleich zu den anderen Ballungsgebieten - höchsten Anteil an selbstgenutztem Wohneigentum. 
Cluster 6 und 7 kennzeichnen stark sozial benachteiligte Bezirke, die in der Vergangenheit durch einen hohen Arbeiteranteil geprägt waren. Sie sind beide in etwa gleich häufig und beschreiben zusammen ca. 20 Prozent aller Bezirke in NRW. Die Arbeitslosenquote liegt bei beiden im Jahr 2011 mit über 12 Prozent weit über dem Landesdurchschnitt. In beiden Clustern ist der Migrantenanteil sehr hoch und der Anteil an selbstgenutztem Wohneigentum sehr niedrig. Trotz dieser offensichtlichen Ähnlichkeiten gibt es aber auch prägnante Unterschiede. So beschreibt Cluster 6 eher alternde Bezirke, die nahezu ausschließlich im Ruhrgebiet liegen; die Einwohnerdichte ist eher rückläufig. Der Jugendquotient ist hier besonders niedrig und weist ebenfalls den stärksten Rückgang auf. Der Ausländeranteil ist von 1970 bis 2011 um ca. 7 Prozentpunkte gewachsen. Cluster 6 beschreibt sozial benachteiligte, eher schrumpfende und alternde Bezirke.

Abb. 1: Clusterverteilung der kleinräumigen Bezirke für die Jahre 1970 und 2011, NRW und Ruhrgebiet

(a) Klassierung der zeitinvarianten kleinräumigen Bezirke für 1970 und 2011 in NRW

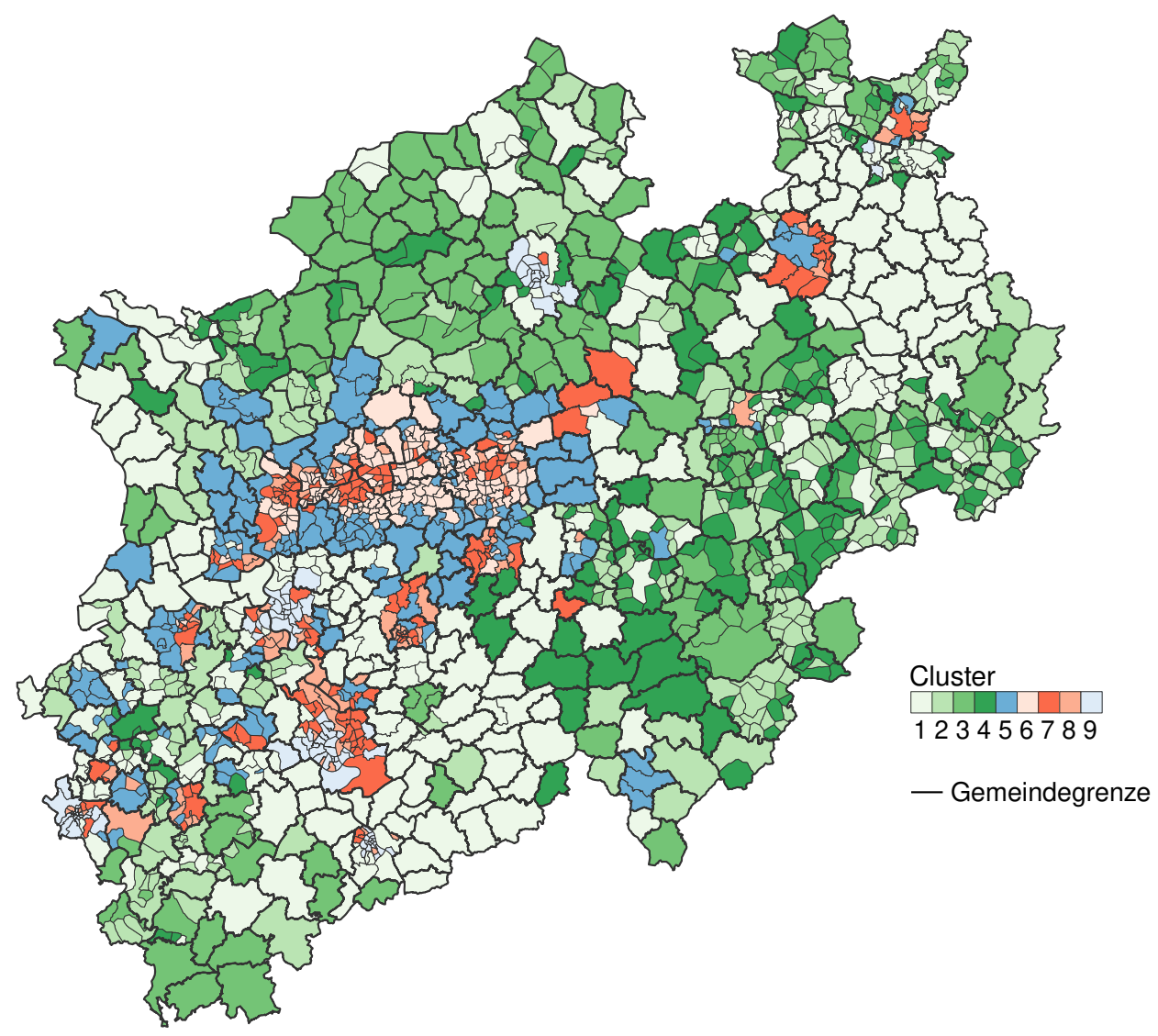




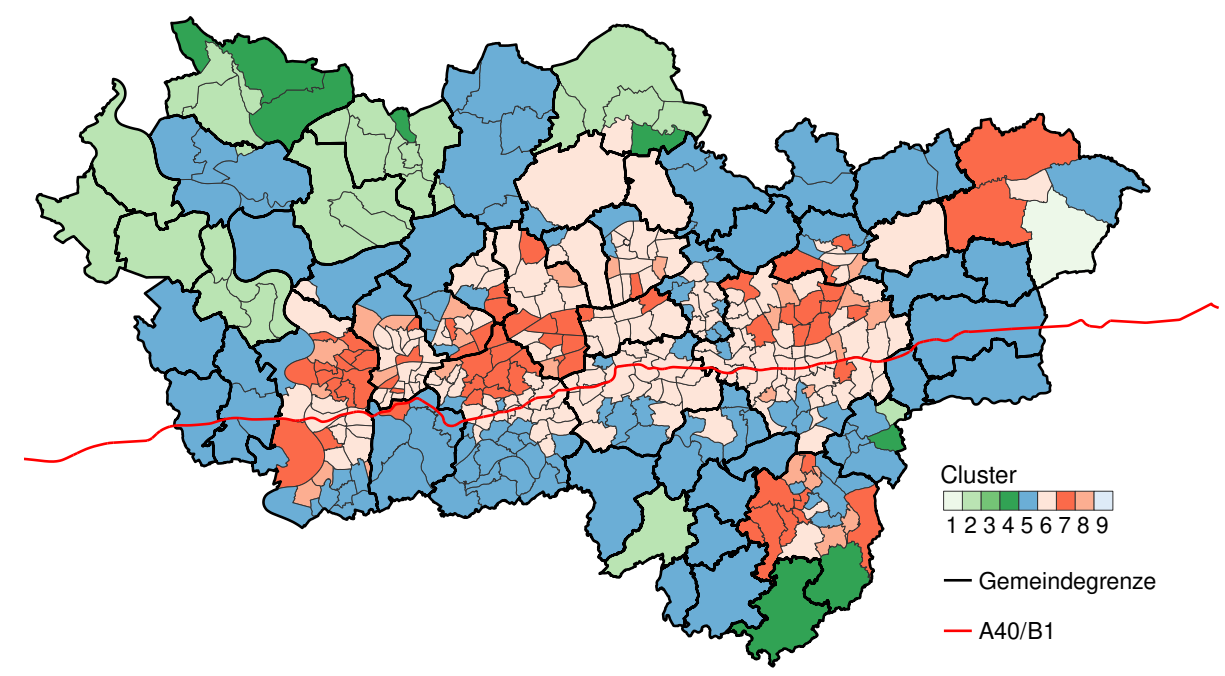

Quelle: Zensus 1970, 2011 und infas 360; eigene Berechnungen

Cluster 7 ist demgegenüber gewachsen, und die Einwohnerdichte ist angestiegen. Der Cluster hat im Mittel den stärksten Zuwachs beim Ausländeranteil; der Migrantenanteil liegt mit knapp 40 Prozent am höchsten; der Jugendquotient liegt über dem Durchschnitt. Über 45 Prozent der Bezirke des Clusters liegen im Ruhrgebiet; dies sind bspw. Bezirke im Duisburger, Essener und Dortmunder Norden sowie im Gelsenkirchener Süden. Man findet solche Bezirke aber auch in Köln, Wuppertal, Hagen und Bielefeld. Cluster 7 beschreibt sozial benachteiligte, wachsende und familienstarke Bezirke.

Cluster 8 ist der kleinste Cluster und enthält nur 5,2 Prozent der kleinräumigen Bezirke. Dieser Cluster kennzeichnet Bezirke in Ballungsregionen mit einem besonders hohen Anteil an Beschäftigten im Produzierenden Gewerbe am Arbeitsort und bildet damit teilweise Gewerbegebiete und angrenzende Wohngebiete ab. Der Rückgang des Produzierenden Gewerbes gegenüber 1970 ist in diesem Cluster gering; der sekundäre Sektor dominiert hier weiterhin. Cluster 8 kann als urbaner Industriecluster bezeichnet werden.

Cluster 9 kennzeichnet vor allem Innenstadtbezirke in Städten außerhalb des Ruhrgebietes, insbesondere in Münster, Düsseldorf, Köln und Bonn sowie Aachen. Im Jahr 2011 waren hier im Mittel über 90 Prozent der Beschäftigten vor Ort im Dienstleistungsbereich tätig. Vor 40 Jahren hatte dieser Cluster auch schon - natürlich auf einem niedrigeren Niveau - den höchsten Dienstleistungsanteil. Diese Bezirke sind strukturell nicht attraktiv für Familien; der Jugendquotient ist am nied- 
rigsten. Man findet hier kaum Leerstände, und der Anteil an Einpersonenhaushalten ist am höchsten. Die Arbeitslosenquote ist im Vergleich $\mathrm{zu}$ den anderen städtischen Bezirken sehr niedrig, der Migrantenanteil nur leicht überdurchschnittlich. Cluster 9 kann als stabiler Dienstleistungscluster bezeichnet werden, der von dem Strukturwandel kaum betroffen ist.

Zusammenfassend zeigt die Längsschnitttypologie, dass sich aus den ehemaligen Arbeiterbezirken vor allem zwei Typen sozial benachteiligter Bezirke entwickelt haben: einerseits eher alternde, schrumpfende Bezirke (Typ 6), andererseits wachsende, familienstarke Bezirke (Typ 7). Beide Typen sind durch eine sehr hohe Arbeitslosenquote und einen hohen (Typ 6) bis sehr hohen (Typ 7) Migrantenanteil geprägt. Gleichzeitig zeigen die Analysen, dass im wohlhabenderen Süden des Ruhrgebiets der Anteil an Kindern besonders gering ist und die Mehrzahl der Kinder somit im sozial benachteiligten Norden aufwächst.

\section{2 Übergangsquoten zu den Gymnasien in den Bezirkstypen}

Um die Zusammenhänge zwischen Sozialraum und Bildungsteilhabe aufzeigen zu können, werden die Ergebnisse der Typisierung nun mit den Übergangsquoten zu den Gymnasien in zeitlicher Perspektive in Verbindung gebracht. In NRW ist die Schulformempfehlung beim Übergang in die weiterführende Schule nicht bindend. Der Zeitraum von 2007 bis 2010 wird nicht dargestellt, da während dieses Zeitraums die Gesetzeslage u.a. verpflichtende Beratungsgespräche bei nur bedingter Eignung der Schüler*innen vorsah und die Berücksichtigung des Elternwillens durch die Schulformempfehlungen eingeschränkt war. Sollte an einer einzelnen Schule die Zahl der Anmeldungen die Aufnahmekapazität übersteigen, wird die Schulformempfehlung in der Regel nicht bei der Aufnahme berücksichtigt. Stattdessen sind dann u.a. Geschwisterkinder an der Schule, die Nähe der Grundschule oder Schulwege relevant (APO-S I $\$ 1$ Abs. 2).

Abbildung 2 zeigt die Entwicklung der Übergangsquoten zum Gymnasium in den zeitinvarianten Bezirken im Ruhrgebiet. Die mit grünen Punkten markierten Bezirke wiesen sowohl im Zeitraum 2003 bis 2006 als auch im Zeitraum 2011 bis 2014 überdurchschnittliche Übergangsquoten auf. Die Referenz ist dabei jeweils die durchschnittliche Übergangsquote der Bezirke auf Landesebene. Die so markierten Bezirke fallen zumeist in den sozialökonomisch konsolidierten Cluster 5. Besonders auffallend ist jedoch die Verteilung der dunkelroten Rauten, die für beide Zeiträume unterdurchschnittliche Übergangsquoten ausweisen. Diese Markierungen finden sich zumeist in den sozial benachteiligten Bezirken in Cluster 6 und 7. 
Abb. 2: Übergangsquoten zum Gymnasium in den Jahren 2003-2006 und 2011-2014 im Ruhrgebiet, Bezirkstypologie im Hintergrund

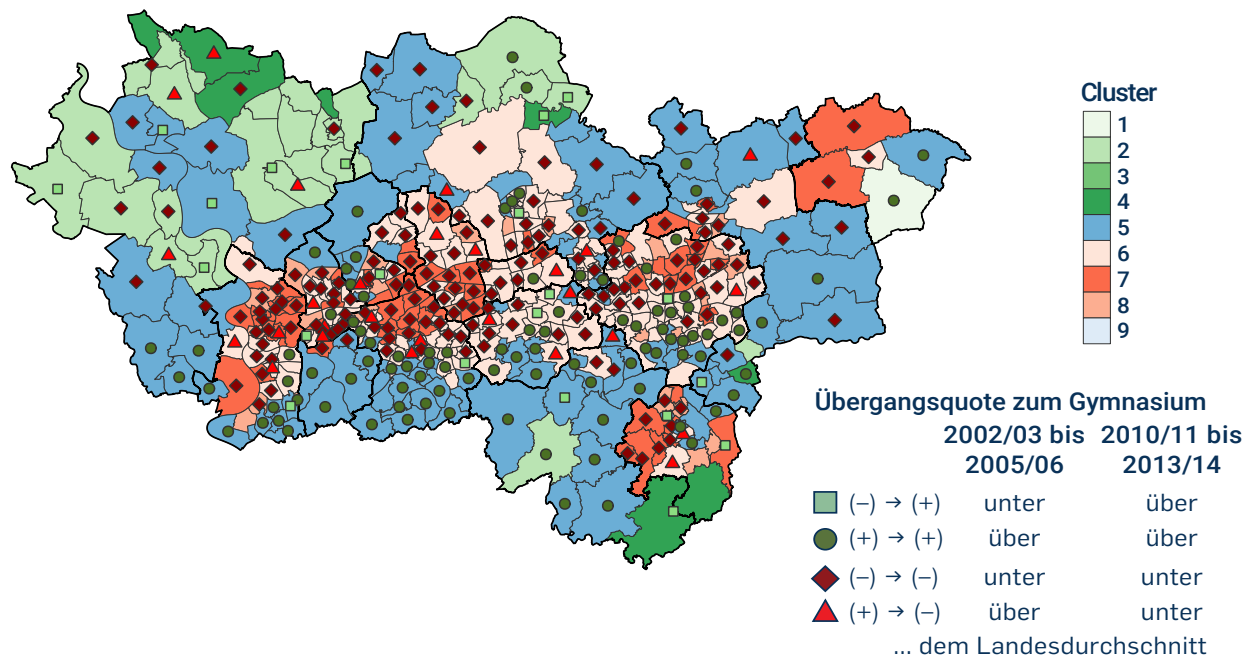

Quelle: IT.NRW, Zensus 1970, 2011 und infas 360; eigene Berechnungen

Die anderen Entwicklungsmöglichkeiten umfassen Veränderungen hin zu unter- oder überdurchschnittlichen Quoten. Sie treten aber insgesamt viel seltener auf und verteilen sich uneinheitlich über die Bezirke in den Clustern.

\subsection{Modellrechnungen für die Übergangsquoten zum Gymnasium}

Mit Hilfe der hier verwendeten amtlichen Daten lassen sich kleinräumige Unterschiede im Sozialraum und in Bezug auf die Bildungssituation nicht nur deskriptiv beschreiben, sondern auch multivariat im Hinblick auf Output-Indikatoren wie die Übergangsquote zum Gymnasium auswerten. Hierzu werden die Übergangsdaten jeweils für die drei Analysezeiträume 2003 bis 2006, 2007 bis 2010 und 2011 bis 2015 gepoolt und jeweils ein lineares Mehrebenenregressionsmodell mit variierenden bezirksspezifischen Konstanten geschätzt. ${ }^{8}$ In dem Modell unterscheiden wir die Schulebene (Level 1) von der Ebene der zeitinvarianten Bezirke (Level 2). Die Verteilung der Übergangsquoten zwischen den Grundschulen ist annähernd normalverteilt, so dass ein lineares Modell angemessen erscheint.

In Anlehnung an Meusburger (1998) werden drei unterschiedliche Einflussfaktoren auf die Übergangsquote berücksichtigt: die Struktur der Schülerschaft, das schulische Angebot und das Umfeld der Schulen. Die Variablen zur Beschreibung der Struktur

8 Ein Mehrebenenmodell wurde gewählt, da zu vermuten ist, dass Schulen innerhalb eines zeitinvarianten Bezirks ähnlicher zueinander sind als Schulen in unterschiedlichen Bezirken und daher die i.i.d.-Annahme verletzt ist. 
der Schülerschaft gehen vornehmlich als Kontrollvariablen in das Modell ein. Die Anzahl der Abgänge beschreibt die Größe der Schule. Der Anteil an Schülerinnen wird kontrolliert, um die tendenziell besseren Schulleistungen und höheren Übergangsquoten auf das Gymnasium von Mädchen mitzuberücksichtigen (Helbig, 2012). Als weitere Kontrollvariable dient der Ausländeranteil an den Abgängen. ${ }^{9}$ Kinder mit nicht deutscher Staatsbürgerschaft wachsen in Haushalten auf, die häufiger von Armut betroffen sind (Ministerium für Arbeit, Integration und Soziales des Landes Nordrhein-Westfalen, 2016, S. 215 ff.), und deren Eltern weisen tendenziell ein geringeres Bildungsniveau auf (Siegert, 2008, S. 46 ff.). Auf individueller Ebene ergibt sich zudem ein schwacher Zusammenhang zwischen dem Migrationshintergrund und dem Schulerfolg (Reiss, Sälzer, Schiepe-Tiska, Klieme \& Köller, 2016, S. 337 ff.).

Im Hinblick auf die Schulwahlentscheidungen, die beim Übergang auf die Sekundarstufe 1 erfolgen, ist auch das Angebot an Gymnasien und Gesamtschulen im Umfeld der Grundschulen relevant (vgl. Sixt, 2010, S. 155). Das lokale Schulangebot an Gymnasien und Gesamtschulen wird zum einen durch die Zahl der Schulen dieser Schulform in den jeweiligen Gemeinden und zum anderen durch die Luftlinienentfernung von der Grundschule zum nächsten Gymnasium bzw. zur nächsten Gesamtschule modelliert. Bei der Grundschulwahl ist die Entfernung zur Schule ein wichtiger Faktor (Riedel, Schneider, Schuchart \& Weishaupt, 2010), und es ist plausibel anzunehmen, dass die Entfernung zwischen der oftmals wohnortnahen Grundschule und der weiterführenden Schule bei der Schulwahl von Bedeutung ist. Um die Stadt-Land-Unterschiede hinsichtlich der Entfernungen zwischen den Schulen zu berücksichtigen, wurde zudem zwischen Groß-, Mittel- und Kleinstädten unterschieden. Sowohl die Anzahl der weiterführenden Schulen als auch die Entfernung zur nächsten Schule werden über die einzelnen Jahre der drei Untersuchungszeiträume gemittelt und gehen als Durchschnittswert in das Modell ein. Es ist $\mathrm{zu}$ vermuten, dass ein großes Schulangebot und örtlich naheliegende Gymnasien die Übergangsquoten zum Gymnasium erhöhen. Eine Gesamtschule in geringer Entfernung zum Wohnumfeld bzw. den Grundschulen kann die Übergänge zum Gymnasium hingegen verringern (vgl. Fickermann, 1999).

Die Bezirkstypologie liefert eine Beschreibung des Umfelds der Grundschulen, die neben der Sozialstruktur der Bezirke auch die historische Entwicklung in diesen Gebieten berücksichtigt. Das Umfeld der Schule kann über das Schulangebot hinaus weiteren Einfluss auf das Übergangsverhalten der Schülerinnen haben. Dabei

9 Der Anteil der Schüler*innen mit Migrationshintergrund wird erst seit dem Jahr 2007 für alle nordrhein-westfälischen Schulen erhoben, so dass für die Analysen in den drei Untersuchungszeiträumen lediglich der Ausländeranteil als Indikator für Schüler*innen mit Zuwanderungsgeschichte genutzt werden kann. Bei der Interpretation des Ausländeranteils an den Übergängen ist zu beachten, dass im Jahr 2009 die ersten Schüler*innen die Grundschule verlassen, die nach der Reform des Staatsangehörigkeitsrechts im Jahr 2000 geboren wurden, und so ein Großteil der Schüler*innen aus Familien mit Zuwanderungsgeschichte, die in Deutschland geboren wurden, nicht mehr von dieser Kategorie erfasst werden. 
kann der Effekt des Sozialraums im Umfeld der Grundschulen auf zwei verschiedene Weisen diskutiert werden: Einerseits liefert die Beschreibung der Bevölkerung im Umfeld der Grundschulen Hinweise über die sozioökonomische Struktur der Schülerschaft. Auf der Ebene der einzelnen Schüler*innen sind Zusammenhänge zwischen sozialem und kulturellem Hintergrund bekannt. Diese individuellen Prozesse und Effekte führen dann zu korrespondierenden Zusammenhängen auf der Makroebene. Das heißt, wenn Kinder aus armen und/oder bildungsfernen Haushalten geringere Chancen auf den Besuch eines Gymnasiums haben, werden auch die Übergangsquoten auf das Gymnasium in den Schulen mit einem hohen Anteil von Kindern aus diesen Haushalten gering sein. Da die amtliche Statistik landesweit keine Daten zum sozioökonomischen Hintergrund der Schülerinnen erhebt, ist der Sozialraum der Grundschulen ein sinnvoller Proxy für die sozioökonomische Struktur der Schülerschaft.

Andererseits wird auch dem Sozialraum selbst ein Effekt auf die individuellen Bildungsverläufe zugeschrieben. Dieser Effekt wird in der Literatur kontroverser diskutiert als die Einflüsse der sozialen Herkunft auf den Gymnasialbesuch und den individuellen Bildungserfolg (Ditton \& Krüsken, 2006, 2007; Goldsmith, 2009; Leckie, 2009; Sykes \& Kuyper, 2009). In der Regel kommen die Untersuchungen zu dem Ergebnis, dass die Effekte der individuellen und familiären Merkmale einen relativ großen Teil der Unterschiede in den Schulleistungen erklären können; darüber hinaus lässt sich jedoch auch ein kleinerer Effekt des Umfeldes feststellen. Da die folgenden Modellrechnungen auf aggregierten Daten basieren, lassen sich die möglichen Einflüsse der Schülerstruktur und des sozialen Umfelds nicht getrennt voneinander schätzen; wir erhalten hier nur den summarischen Einfluss aus Schülerstruktur und Kontexteffekt.

Die Ergebnisse der Regressionsrechnung sind in Tabelle 2 dargestellt. Das Modell für den aktuellsten Zeitraum (2011 bis 2014) wird schrittweise aufgebaut (Modelle 1.1-1.3); das vollständige Modell 1.3 wird danach auch für die beiden weiter zurückliegenden Beobachtungszeiträume geschätzt. Im einfachsten Modell 1.1 wird zunächst nur der Anteil nicht deutscher Schüler*innen kontrolliert; hier liegt die Intraklassenkorrelation mit 0,57 relativ hoch, d.h., dass knapp 60 Prozent der Varianz in den Gymnasialquoten auf die Variation zwischen den Bezirken zurückzuführen sind. Dieser Anteil reduziert sich mit Hinzunahme weiterer Variablen. Modell 1.2 enthält zusätzliche Variablen zum Schulangebot, was zu einer Reduktion der Intraklassenkorrelation auf 0,44 führt. In dem Modell 1.3 werden eine ganze Reihe weitere Kontextvariablen einbezogen; die Intraklassenkorrelation sinkt dann auf 0,38. Ein Maß für die Güte des Modells ist die erklärte Varianz der Übergangsquote. Sie liegt in Modell 1.3 bei 41 Prozent, in den korrespondierenden Modellen 2.3 und 3.3 für die früheren Zeiträume sogar bei 49 Prozent und 47 Prozent. Dies bedeutet, dass knapp 50 Prozent der Varianz der Übergangsquote durch die Modellvariablen erklärt werden können. 
Tab. 2: $\quad$ Mehrebenenregressionsmodell für die Übergangsquote zum Gymnasium im Zeitraum 2011-2014

\begin{tabular}{|c|c|c|c|c|c|}
\hline \multirow[b]{3}{*}{ Modell } & \multicolumn{5}{|c|}{ Übergangsquote zum Gymnasium im Zeitraum } \\
\hline & \multicolumn{3}{|c|}{2011 bis 2014} & \multirow{2}{*}{$\begin{array}{c}2007 \text { bis } \\
2010 \\
2.3\end{array}$} & \multirow{2}{*}{$\begin{array}{c}2003 \text { bis } \\
2006 \\
3.3\end{array}$} \\
\hline & 1.1 & 1.2 & 1.3 & & \\
\hline Abgänge (Anzahl) & $0,01^{* *}$ & $0,01^{*}$ & $0,01^{* *}$ & 0 & 0 \\
\hline davon weiblich (\%) & $0,12^{*}$ & $0,11^{*}$ & $0,10^{*}$ & 0,07 & 0,05 \\
\hline davon Ausländer (\%) & $-1,15^{\star \star}$ & $-1,37^{\star *}$ & $-1,30^{\star *}$ & $-0,85^{\star *}$ & $-0,74^{\star *}$ \\
\hline Distanz Gesamtschule (km) & & $0,12^{*}$ & $0,19^{\star *}$ & 0,01 & $-0,01$ \\
\hline Distanz Gymnasium (km) & & $-1,14^{* *}$ & $-1,01^{* *}$ & $-1,01^{* *}$ & $-1,02^{* *}$ \\
\hline Anz. Gymnasien in der Gemeinde & & $0,81^{* *}$ & $0,47^{* *}$ & $0,40^{* *}$ & $0,43^{*}$ \\
\hline Anz. Gesamtschulen in der Gem. & & $-0,59^{* *}$ & $-0,08$ & $-0,09$ & $-0,18$ \\
\hline Cluster 1 (Referenz: Cluster 5) & & & $-0,10$ & $-0,67$ & 0,14 \\
\hline Cluster 2 & & & $-2,26$ & $-1,82$ & $-1,95$ \\
\hline Cluster 3 & & & $-2,84^{*}$ & $-4,37^{* *}$ & $-3,74^{* *}$ \\
\hline Cluster 4 & & & $-3,05^{*}$ & $-3,64^{* *}$ & $-3,47^{*}$ \\
\hline Cluster 6 & & & $-7,84^{\star *}$ & $-5,56^{\star \star}$ & $-5,04^{\star *}$ \\
\hline Cluster 7 & & & $-10,92^{* *}$ & $-7,27^{* *}$ & $-6,50^{* *}$ \\
\hline Cluster 8 & & & $-6,71^{* *}$ & $-4,77^{* *}$ & $-4,49^{* *}$ \\
\hline Cluster 9 & & & $5,20^{* *}$ & $5,66^{* *}$ & $5,27^{* *}$ \\
\hline Mittelstadt (Referenz: Kleinstadt) & & & 0,62 & 0,66 & 0,61 \\
\hline Großstadt & & & $6,06^{\star *}$ & $5,73^{* *}$ & $4,62^{* *}$ \\
\hline Konstante & $38,74^{\star \star}$ & $40,52^{* *}$ & $40,66^{* *}$ & $43,57^{* *}$ & $43,25^{\star *}$ \\
\hline $\mathrm{R}^{2}$ & 0,124 & 0,336 & 0,411 & 0,49 & 0,473 \\
\hline ICC & 0,571 & 0,444 & 0,381 & 0,46 & 0,395 \\
\hline $\mathrm{N}$ & 2.747 & 2.747 & 2.747 & 2.747 & 2.747 \\
\hline
\end{tabular}

Anm.: $\quad{ }^{*} \mathrm{p}<0.05 ;{ }^{* *} \mathrm{p}<0.01$.

Quelle: IT.NRW, Zensus 1970 und 2011; eigene Berechnungen

Die Größe und Richtung der geschätzten Koeffizienten der Modelle 1.3, 2.3 und 3.3 lassen sich nun miteinander vergleichen. So weist der Anteil der ausländischen Schüler ${ }^{\star}$ innen über alle drei Zeiträume hinweg an den Abgängen einen negativen Effekt auf die Übergangsquote auf. Dieser Effekt nimmt mit der Zeit zu und ist im letzten Untersuchungszeitraum 2011 bis 2014 mit -1,30 fast doppelt so groß wie im Zeitraum 2003 bis 2006 (-0,74). Diese Zunahme kommt vermutlich dadurch zustande, dass im letzten Zeitraum nur noch Kinder mit eigener Zuwanderungsgeschichte durch das Merkmal erfasst werden und diese deutlich seltener auf ein Gymnasium wechseln, wohingegen im ersten Zeitraum teilweise auch die Kinder der zweiten und dritten Einwandergeneration miterfasst wurden. Der Einfluss des Anteils der 
Mädchen ist nur im letzten Zeitraum signifikant ${ }^{10}$ und wirkt sich positiv auf die Übergangsquote aus.

Es zeigt sich, dass sowohl die Distanz zum Gymnasium und zur Gesamtschule als auch die Anzahl der Schulen dieser Schulform für alle Zeiträume einen signifikanten Effekt auf die Übergangsquote zum Gymnasium aufweisen: Mit weiterer Entfernung von der Grundschule zum nächsten Gymnasium sinkt die Übergangsquote, wohingegen die Übergangsquote zum Gymnasium mit der Entfernung zur nächsten Gesamtschule steigt. ${ }^{11}$ Die Zahl der Gymnasien hat einen positiven Effekt auf die Übergangsquote, wohingegen die Zahl der Gesamtschulen einen negativen Effekt hat. Je größer das Angebot an Gymnasien ist, desto eher finden auch Übergänge zum Gymnasium statt. Da Gesamtschulen seltener sind als Gymnasien und in vielen Gemeinden gar keine Gesamtschulen vorhanden sind, ist der Effekt so zu interpretieren, dass sich bei einer Wahlmöglichkeit der Eltern zwischen Gymnasium und Gesamtschule die Übergangsquote zum Gymnasium leicht reduziert. Im Zeitablauf nimmt dieser Effekt jedoch etwas ab.

Für die Bezirke und Gemeinden, in denen die Grundschulen liegen, wurden einige relevante Kontextmerkmale berücksichtigt. So sind die Übergangsquoten zum Gymnasium in den Großstädten im Zeitraum 2011 bis 2014 durchschnittlich 6 Prozentpunkte höher als in den Kleinstädten.

Die Effekte der verschiedenen Entwicklungscluster werden jeweils im Vergleich zu dem sozialökonomisch konsolidierten, eher bürgerlichen Cluster 5 angegeben. Hier zeigt sich auch unter Kontrolle von Schulangebot und Struktur der Abgänge, dass es deutliche Unterschiede zwischen den Grundschulen in den Bezirken gibt. Die beiden Cluster 6 und 7 kennzeichnen sozial besonders benachteiligte Bezirke. Im jüngsten Untersuchungszeitraum weisen sie mit -7,8 bzw. -10,9 Prozentpunkten zusammen mit den Bezirken im urbanen Industriecluster 8 mit -6,7 Prozentpunkten die geringsten Übergangsquoten im Vergleich zu Schulen in den konsolidierten Bezirken des Clusters 5 auf. $^{12}$ Im Vergleich der Modelle für die drei Zeitpunkte ist zu erkennen, dass die Effektstärke für die Clusterzugehörigkeit sogar zugenommen hat. So erhöht sich der Abstand in der Übergangsquote für die Schulen im Cluster 7 von 6,5 Prozentpunkten im Zeitraum 2003 bis 2006 auf 7,3 Prozentpunkte im Zeitraum 2007

10 Da es sich bei dem vorliegenden Datensatz um eine Vollerhebung handelt, können die Standardfehler und Signifikanzen nicht als Ausdruck eines Stichprobenfehlers interpretiert werden.

11 Im Modell für den Zeitraum 2003 bis 2006 ist der Koeffizient für die Distanz zur Gesamtschule negativ. Im nächsten Modell ist der Effekt jedoch bereits nicht mehr signifikant und wechselt bei den späteren Zeiträumen das Vorzeichen. Vermutlich spiegeln sich hier die geringeren Übergangsquoten der Grundschulen in den ländlichen Bezirken wider, die weitere Entfernungen zur nächsten Gesamtschule aufweisen.

12 Wird zusätzlich für die Schüler*innenzusammensetzung an den einzelnen Schulen kontrolliert, bleiben diese Effekte bestehen, werden aber geringfügig schwächer (vgl. Jeworutzki et al., 2017, S. 190). 
bis 2010 und erreicht mit 10,9 Prozentpunkten den höchsten Wert im Zeitraum 2011 bis 2014. Dies ist ein deutlicher Hinweis auf eine kontinuierliche Zunahme der kleinräumigen Bildungsdisparitäten. Eine Ausnahme bilden die Grundschulen im „stabilen Dienstleistungscluster" 9, der aber im Ruhrgebiet gar nicht vorkommt. Diese Schulen weisen in allen drei Zeitpunkten noch einmal deutlich höhere Übergangsquoten auf als die im konsolidierten bürgerlichen Cluster 5.

\section{Fazit}

Die vorliegende Studie ist ein Beispiel für ein flächendeckendes überregionales kleinräumiges Bildungsmonitoring auf Basis von amtlichen Daten, welches sowohl die Entwicklung der Sozialstruktur als auch der Bildungslandschaft unterhalb der Gemeindeebene in NRW zusammen betrachtet. Dabei wurden die Bezirke im Gebiet des Ruhrgebiets besonders in den Blick genommen und sowohl regionale als auch kleinräumige Disparitäten in der Bildungsteilhabe untersucht.

Inhaltlich verweisen unsere deskriptiven und multivariaten Analyseergebnisse auf einen starken Zusammenhang zwischen dem Sozialraum der Grundschulen und den Übergangsquoten. Wenn die Grundschulen in Bezirken liegen, deren Entwicklung im Verlauf des Strukturwandels von Arbeitervierteln hin zu sozial benachteiligten Bezirken erfolgte, sind die Teilhabechancen der Kinder deutlich geringer als etwa in konsolidierten bürgerlichen Bezirken. Dabei zeigen die Modelle, dass der Abstand zwischen diesen beiden Bezirkstypen erheblich ist. Bedenklich ist zudem, dass bei der Betrachtung der drei untersuchten Zeiträume die Spanne des Gymnasialübergangs zwischen den Grundschulen in den benachteiligten Bezirken und den bürgerlichen Bezirken über die Jahre zugenommen hat.

Besonders problematisch ist dieser Befund, weil bereits das Ausgangsniveau der Grundschulen in den sozial benachteiligten Bezirken sehr viel niedriger war. Während also tendenziell mehr Kinder aus den bürgerlichen Bezirken auf die Schulform wechseln, die am unmittelbarsten die Zugangsmöglichkeit zur akademischen Bildung eröffnet, fallen die Kinder aus den benachteiligten Bezirken zurück.

Aus den Untersuchungsergebnissen zum Einfluss des Sozialraums auf die Bildungsbeteiligung folgt für die bildungspolitische Diskussion, dass der Sozialraum und die Struktur der Schülerschaft an den Schulen nicht nur bei der Auswertung von Lernstandsergebnissen (Schräpler \& Jeworutzki, 2016), sondern bei der Förderung der Schulen auch eine stärkere Berücksichtigung finden sollten (Möller \& Bellenberg, 2017; Weishaupt, 2016). Eine Förderung der Schulen alleine wird jedoch nicht ausreichen, um die Bildungslücke zwischen den sozial benachteiligten Bezirken und den bürgerlichen Quartieren zu verringern. Die Bemühungen um eine chancengerechte 
Kleinräumiges regionales Bildungsmonitoring |

Bildungslandschaft müssen an eine sozialraumorientierte Sozialpolitik gekoppelt werden, um die Lebensverhältnisse in den benachteiligten Quartieren nachhaltig zu verbessern (El-Mafaalani \& Kurtenbach, 2015).

Die Studie zeigt, dass auf Basis von kleinräumigen amtlichen Zensusdaten auch ein überregionales Monitoring möglich ist. In Zukunft werden (wie schon beim Zensus 2011) zumindest die Zensusdaten von den Statistischen Ämtern georeferenziert und auf einer Rasterebene in anonymisierter Form veröffentlicht, so dass diese Kontextmerkmale in ein überregionales kleinräumiges Bildungsmonitoring miteinfließen können.

\section{Literatur und Internetquellen}

Autorengruppe Bildungsberichterstattung (Hrsg.). (2018). Bildung in Deutschland 2018. Ein indikatorengestützter Bericht mit einer Analyse zu Wirkungen und Erträgen von Bildung. Bielefeld: wbv.

Baethge, M., Solga, H., \& Wieck, M. (2007). Berufsbildung im Umbruch. Signale eines überfälligen Aufbruchs. Berlin: Friedrich-Ebert-Stiftung.

Bartels, J. (1975). Interdependenzen zwischen dem Angebot und der Nachfrage nach Plätzen der Sekundarschulstufe bei Angehörigen unterschiedlicher Schichtzugehörigkeit in einer Großstadt. Berlin: Technische Universität Berlin.

Baumert, J., Maaz, K., Gresch, C., McElvany, N., Anders, Y., Jonkmann, K., Neumann, M., \& Watermann, R. (2010). Der Übergang von der Grundschule in die weiterführende Schule - Leistungsgerechtigkeit und regionale, soziale und ethnisch-kulturelle Disparitäten: Zusammenfassung der zentralen Befunde. In K. Maaz, J. Baumert, C. Gresch \& N. McElvany (Hrsg.), Der Übergang von der Grundschule in die weiterführende Schule - Leistungsgerechtigkeit und regionale, soziale und ethnisch-kulturelle Disparitäten (S. 5-21). Berlin: BMBF.

Bundesministerium für Bildung und Forschung (Hrsg.). (2017). Berufsbildungsbericht 2017. Berlin: BMBF.

Csardi, G., \& Nepusz, T. (2006). The Igraph Software Package for Complex Network Research. InterJournal Complex System, 1695.

Ditton, H., \& Krüsken, J. (2006). Sozialer Kontext und schulische Leistungen: zur Bildungsrelevanz segregierter Armut. Zeitschrift für Soziologie der Erziehung und Sozialisation, 26, 135-157.

Ditton, H., \& Krüsken, J. (2007). Sozialräumliche Segregation und schulische Entwicklung. Diskurs Kindheits- und Jugendforschung, (2), 23-38.

Döbert, H. (2007). Indikatorenkonzept und Beschreibung von Beispielindikatoren für eine regionale Bildungsberichterstattung. Gütersloh: Bertelsmann Stiftung.

El-Mafaalani, A., \& Kurtenbach, S. (2015). Das Raumparadoxon der Bildungspolitik. Warum Bildungsinvestitionen sozialräumlicher Segregation nicht entgegenwirken. In A. El-Mafaalani, S. Kurtenbach \& K. P. Strohmeier (Hrsg.), Auf die Adresse kommt es an. Segregierte Stadtteile als Problem- und Möglichkeitsräume begreifen. Weinheim \& Basel: Beltz Juventa.

Fickermann, D. (1999). Grundschulzeugnis und Schulformwahlverhalten. Untersuchungen zur Leistungsselektivität beim Übergang in die Sekundarstufe I. In H. Weishaupt (Hrsg.), Zum Übergang auf weiterführende Schulen. Statistische Analysen und Fallstudien (S. 113-155). Erfurt: Pädagogische Hochschule. 
Goldsmith, P. R. (2009). Schools or Neighborhoods or Both? Race and Ethnic Segregation and Educational Attainment. Social Forces, 87, 1913-1941. https://doi.org/10.1353/ sof.0.0193

Hauf, T. (2007). Innerstädtische Bildungsdisparitäten an der Übergangsschwelle von den Grundschulen zum Sekundarschulsystem. Zeitschrift für Pädagogik, 53, 299-313.

Helbig, M. (2012). Sind Mädchen besser? Der Wandel geschlechtsspezifischen Bildungserfolgs in Deutschland. Frankfurt a. M.: Campus.

Hetmeier, H.-W., Schneider, C., Wolf, R., Klostermann, T., Gnahs, D., \& Weiß, C. (2014). Anwendungsleitfaden zum Aufbau eines kommunalen Bildungsmonitorings. Wiesbaden, Stuttgart \& Bonn: DIE space.

Jeworutzki, S., \& Knüttel, K. (2018). Empirische Bildungslandschaften: Netzwerke verstetigter Übergangsbeziehungen zwischen Grund- und weiterführenden Schulen. Stadtforschung und Statistik: Zeitschrift des Verbandes Deutscher Städtestatistiker, 31 (1), 3541.

Jeworutzki, S., Knüttel, K., Niemand, C., Schmidt, B.-J., Schräpler, J.-P., \& Terpoorten, T. (2017). Räumlich segregierte Bildungsteilhabe in NRW und im Ruhrgebiet. In J.-P. Schräpler, S. Jeworutzki, B. Butzin, T. Terpoorten, J. Goebel \& G. G. Wagner (Hrsg.), Wege zur Metropole Ruhr (S. 11-213). Bochum: Zefir.

KMK (Sekretariat der Ständigen Konferenz der Kultusminister der Länder in der Bundesrepublik Deutschland) (Hrsg.). (2016). Gesamtstrategie der Kultusministerkonferenz zum Bildungsmonitoring. Berlin \& Bonn: KMK.

Landeshauptstadt München (Hrsg.). (2016). Münchner Bildungsbericht 2016. München: Landeshauptstadt.

Leckie, G. (2009). The Complexity of School and Neighbourhood Effects and Movements of Pupils on School Differences in Models of Educational Achievement. Journal of the Royal Statistical Society: Series A (Statistics in Society), 172, 537-554. https://doi. org/10.1111/j.1467-985x.2008.00577.x

Meusburger, P. (1998). Bildungsgeographie. Wissen und Ausbildung in der räumlichen Dimension. Heidelberg \& Berlin: Spektrum Akademischer Verlag.

Ministerium für Arbeit, Integration und Soziales des Landes Nordrhein-Westfalen (Hrsg.). (2016). Sozialbericht NRW 2016. Armuts- und Reichtumsbericht. Düsseldorf: Ministerium.

Möller, G., \& Bellenberg, G. (2017). Ungleiches ungleich behandeln. Standortfaktoren berücksichtigen - Bildungsgerechtigkeit erhöhen - Bildungsarmut bekämpfen. Essen: Neue Deutsche Schule.

NRW.ProjektSoziales GmbH (Hrsg.). (2017). Strategische Sozialplanung. FSA-Infogramm.

Peisert, H. (1967). Soziale Lage und Bildungschancen in Deutschland. München: Piper.

Regionalverband Ruhr (Hrsg.). (2012). Bildungsbericht Ruhr. Münster: Waxmann.

Reiss, K., Sälzer, C., Schiepe-Tiska, A., Klieme, E., \& Köller, O. (Hrsg.). (2016). PISA 2015: eine Studie zwischen Kontinuität und Innovation. Münster et al.: Waxmann.

Riedel, A., Schneider, K., Schuchart, C., \& Weishaupt, H. (2010). School Choice in German Primary Schools. How Binding Are School Districts? Journal for Educational Research Online, 2 (1), 94-120.

Schräpler, J.-P., \& Jeworutzki, S. (2016). Der Sozialindex für NRW - Die Bildung von Schulstandorttypen über SGB-II-Dichten und Migrationshintergrund. In B. GrootWilken, K. Isaac \& J.-P. Schräpler (Hrsg.), Sozialindices für Schulen. Hintergründe, Methoden und Anwendung. Münster et al.: Waxmann.

Schräpler, J.-P., Jeworutzki, S., Butzin, B., Terpoorten, T., Goebel, J., \& Wagner, G. G. (Hrsg.). (2017). Wege zur Metropole Ruhr. Bochum: Zefir.

Siegert, M. (2008). Schulische Bildung von Migranten in Deutschland. Nürnberg: Bundesamt für Migration und Flüchtlinge. 
Sixt, M. (2010). Regionale Strukturen als herkunftsspezifische Determinanten von Bildungsentscheidungen. Dissertation. Universität Kassel.

Stadt Dortmund (Hrsg.). (2014). Zweiter kommunaler Bildungsbericht der Stadt Dortmund. Dortmund: Stadt Dortmund, Fachbereich Schule.

Stadt Essen (Hrsg.). (2011). Der Bildungsbericht 2011. Essen: Stadt Essen.

Sykes, B., \& Kuyper, H. (2009). Neighbourhood Effects on Youth Educational Achievement in the Netherlands: Can Effects Be Identified and Do They Vary by Student Background Characteristics? Environment and Planning A, 41, 2417-2436. https://doi. org/10.1068/a41255

Terpoorten, T. (2014). Räumliche Konfiguration der Bildungschancen: Segregation und Bildungsdisparitäten am Übergang in die weiterführenden Schulen im Agglomerationsraum Ruhrgebiet. Bochum: ZEFIR.

Weishaupt, H. (2016). Schulen in schwieriger Lage und Schulfinanzierung. Die Deutsche Schule, 108 (4), 354-369.

Sebastian Jeworutzki, geb. 1981, wissenschaftlicher Mitarbeiter in der Fakultät für Sozialwissenschaft der Ruhr-Universität Bochum.

E-Mail: sebastian.jeworutzki@ruhr-uni-bochum.de

Jörg-Peter Schräpler, Prof. Dr., geb. 1961, Professor für sozialwissenschaftliche Datenanalyse an der Ruhr-Universität Bochum.

E-Mail: joerg-peter.schraepler@ruhr-uni-bochum.de

Anschrift: Ruhr-Universität Bochum, Universitätsstraße 150, 44801 Bochum 